\title{
Profiles
}

\section{Chengye Sun, China CDC's Chief Expert of Poison Control}

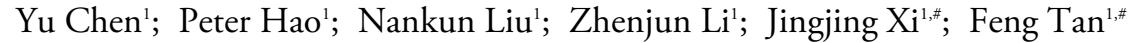

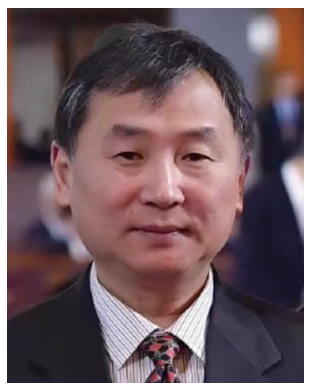

In July 1985, Chengye Sun graduated from Henan Medical University and became a physician in a hospital. In 1988, he entered the Chinese Academy of Preventive Medical Sciences for postgraduate studies on occupational medicine, which helped him become a public health professional. The shift from being a clinician to a public health worker helped him realize how external factors could act as strong drivers on human health.

In 1993, Sun noticed that the diversity of poisons and the complexity of people's exposure to poisons were far beyond the ability of physicians to control as even experts in this field had difficulty providing appropriate treatments for poisoned patients, and disposal plans for poisons were based on personal perspectives. To address this issue, Sun applied information technology to collating and analyzing toxicological data with his colleagues. They developed toxicological databases and data sharing platforms, which greatly facilitated poison treatment and poison research. The toxicology database has been under continuous development since the mid-1990s. At present, more than 2,000 hospitals or professional medical institutions have been included in this platform. It has become the world's largest Chinese toxicology database with users all over the country.

With the expansion of poison control tasks, the Poison Control Center of the Chinese Academy of Preventive Medical Sciences was established in January 1999. Sun has contributed greatly to the establishment and effective operation of this center. The poison control center has been in operation for 22 years, providing 24/7 telephone and Internet-based services to medical professionals and the public across the country.

After the severe acute respiratory syndrome (SARS) epidemic in 2003, China's public emergency management has attracted more and more attention. As a domestic expert on poison control, Sun has participated in drafting and modifying several relevant laws, regulations, and policies, and emergency poisoning accidents were included in the category of public health emergencies.

Since 2005, the Poison Control Center of China CDC has undertaken the task of building a national level chemical poisoning treatment base and emergency response team for sudden poisoning incidents and coordinating the national professional forces, and is responsible for the organization and technical guidance of national poisoning health emergency work. As the leader of poison control, Sun has devoted himself to the national health emergency work for poisoning such as the establishment of national remote consultation system for emergency poisoning in 2008, the drafting of the Health emergency plan of the Ministry of Health for poisoning emergencies and Health Emergency Response Technical Plan for Acute Poisoning on 14 Types of Common Toxicants in 2011, the exploration of network service models on health emergency work for poisoning and the Platform of Health Emergency for Poisoning in 2012, etc.

In addition to the great contribution to poison information and emergency management, Sun has also been engaged in poison control research. In the mid-1990s, several "sudden deaths of unknown causes" occurred in many provinces in China. After a series of field investigations, experiments, and clinical studies led by Sun, the disease was confirmed to be caused by the highly toxic rodenticide tetramine. Sun's team then developed rapid diagnostic methods and treatment plans and promoted the banning of tetramine at the national level. With their effort, tetramine poisonings were brought under control. The case was compiled into textbooks, and the research won the Science and Technology Award of the Chinese Preventive Medical Association.

After 2000, Sun noticed that the frequent occurrence and high mortality of mushroom poisonings became a public health problem in some areas of China. He carried out difficult exploration and research and established a toxic biological specimen bank in China CDC, which has now become the largest poisonous biological entity specimen bank in China. United with experts in public health, clinical medicine, biological identification, and other fields, he established a network work model and carried out a series of studies on toxins detection methods, 
pathogenesis, toxicology, and standard clinical therapy of mushroom poisoning prevention and control. Currently, China is a leader in mushroom poisoning prevention and control worldwide.

Being highly recognized in the field of poison control, Professor Sun is also known as a member of the Public Health Emergencies Expert Group of the China National Health Commission (NHC) (Leader of Poison Disposal Team), expert of the World Health Organization (WHO) International Health Regulations on Chemical Safety, Advisory Expert of National Chemical Terrorist Incident Response Team, an Expert of National Food Safety Risk Assessment Expert Committee, the Director of Chinese Preventive Medical Association, the Chair of Occupational Disease Committee of Chinese Preventive Medical Association, Director of Chinese Medical Association, and the Deputy Leader of Toxicology Group of Chinese Medical Association for Emergency, etc.

China is now in a period of rapid development, and Sun had maintained his focus on the poisoning rescue in chemical industrial parks in the past three years. He will continue to devote himself to promote production safety, public health, and social development.

doi: $10.46234 / \mathrm{ccdcw} 2021.106$

\# Corresponding authors: Jingjing Xi, xijj@chinacdc.cn; Feng Tan, tanfeng@chinacdc.cn.

${ }^{1}$ Chinese Center for Disease Control and Prevention, Beijing, China.

Submitted: April 27, 2021; Accepted: April 29, 2021 